\title{
Rates of phenotypic evolution of ecological characters and sexual traits during the Tanganyikan cichlid adaptive radiation
}

\author{
A. GONZALEZ-VOYER*† \& N. KOLM* \\ *Department of Animal Ecology, Evolutionary Biology Centre, Uppsala University, Uppsala, Sweden \\ †Department of Integrative Ecology, Estación Biológica de Doñana (EBD-CSIC), Sevilla, Spain
}

\section{Keywords:}

adaptation;

habitat;

phenotypic evolution;

rate of evolution;

sexual dimorphism;

Tanganyikan cichlid.

\begin{abstract}
Theory suggests that sexual traits evolve faster than ecological characters. However, characteristics of a species niche may also influence evolution of sexual traits. Hence, a pending question is whether ecological characters and sexual traits present similar tempo and mode of evolution during periods of rapid ecological divergence, such as adaptive radiation. Here, we use recently developed phylogenetic comparative methods to analyse the temporal dynamics of evolution for ecological and sexual traits in Tanganyikan cichlids. Our results indicate that whereas disparity in ecological characters was concentrated early in the radiation, disparity in sexual traits remained high throughout the radiation. Thus, closely related Tanganyikan cichlids presented higher disparity in sexual traits than ecological characters. Sexual traits were also under stronger selection than ecological characters. In sum, our results suggest that ecological characters and sexual traits present distinct evolutionary patterns, and that sexual traits can evolve faster than ecological characters, even during adaptive radiation.
\end{abstract}

\section{Background}

Adaptive radiation refers to groups of organisms that have exhibited exceptionally rapid adaptive diversification into a variety of ecological niches (Schluter, 2000; Gavrilets \& Losos, 2009). This process is potentially responsible for much of the phenotypic diversity of life on earth (Simpson, 1953). Adaptive radiation comprises two main components: the creation of new species and the adaptation of constituent species to a diversity of ecological niches. Although often associated with a high rate of speciation, this is not a necessary prerequisite as some well-known adaptive radiations have relatively low species richness (e.g. Darwins finches; Gavrilets \& Losos, 2009). The classical view of adaptive radiation places the main focus on ecological opportunity, in which an ancestral species finds itself in an environment where resources are either abundant or underutilized (Schluter, 2000). Intrinsic characteristics of the organism enable it

Correspondence: Alejandro Gonzalez-Voyer, Department of Animal Ecology, Evolutionary Biology Centre, Uppsala University, Norbyvä gen 18D, Uppsala, 75236, Sweden.

Tel.: +34 954 466700, ext. 1749; fax: +34 954 621125;

e-mail: alejandro.gonzalez@ebd.csic.es to flourish in this resource-rich environment (Liem, 1973; Schluter, 2000). Hence, extrinsic factors provide the opportunity and intrinsic factors the potential for radiation (Schluter, 2000; Gavrilets \& Losos, 2009). Key innovations, such as the pharyngeal apophysis of cichlid fishes (Liem, 1973; Hulsey et al., 2006; Mabuchi et al., 2007), may provide some organisms with a competitive edge in the battle over limited resources. The pharyngeal jaws of cichlids are but one example of a suite of morphological adaptations to the exploitation of different resources during adaptive radiation (e.g. Schluter, 2000; Bouton et al., 2002; Losos \& Miles, 2002; Clabaut et al., 2007; Foster et al., 2007). Hence, under the classical view of adaptive radiation, phenotypic disparity mainly results from divergent natural selection.

However, accumulating evidence highlights the potentially important role that sexual selection can play in generating phenotypic divergence resulting in reproductive isolation and speciation (e.g. Deutsch, 1997; Seehausen et al., 1999; Seehausen \& Van Alphen, 1999; Seehausen, 2000; Boake, 2005; Seehausen et al., 2008). Some of the most compelling evidence comes from studies of haplochromine cichlids. The highly elaborate reproductive behaviours of cichlids have been suggested 
as a potential key innovation favouring diversification (Crapon de Caprona, 1986; Barlow, 2000), and sexual selection through mate choice has been suggested as the main force driving the evolution of colour diversity of haplochromine cichlids in Lakes Malawi and Victoria. The evolution of male coloration is generally preceded by the evolution of a polygynous mating system, and in such polygynous clades, changes in hue occur in frequent association with speciation events (Seehausen et al., 1999). A comparison of nine Malawi cichlids from four genera showed that closely related species differed primarily in colour pattern (Albertson et al., 1999). In Lake Victoria, closely related rock-dwelling cichlid species with fully overlapping geographical distribution almost always differ in sexual dichromatism. Such differences in fin coloration have been suggested to be sufficient to maintain reproductive isolation (Seehausen et al., 1997; Seehausen, 2000). Furthermore, many sympatric species present broad overlap in micro distribution, feeding behaviour and diet (Seehausen, 2000), suggesting that barriers to gene flow are not the result of ecological divergence. Mate choice appears to be the mechanism responsible for reproductive isolation in closely related species as mating is highly assortative even though there is no temporal or spatial isolation of reproductive activities (Seehausen, 2000; Seehausen et al., 2008).

In sum, there is evidence for a key role of both natural and sexual selection in generating phenotypic divergence and diversification during adaptive radiation. A pending question is whether phenotypic traits associated with ecological adaptation, under natural selection, and traits associated with mating success, under sexual selection, present equivalent tempo and mode of evolution during adaptive radiation. On the one hand, verbal arguments, formal mathematical models and available empirical evidence all indicate that sexual characters should exhibit faster evolutionary rates than naturally selected traits (Fisher, 1930; West-Eberhard, 1983; Andersson, 1994; Holland \& Rice, 1998; Gavrilets, 2000). However, it is still unclear whether this is the case during adaptive radiation, when divergence in ecological characters under natural selection proceeds at a rapid pace (Schluter, 2000). On the other hand, opposing natural selection might constrain the evolution of sexual characters (Andersson, 1994). There are also several classic examples of evolutionary change in contemporary timescales driven by natural selection such as beak morphology in Darwin's finches (Grant \& Grant, 2002), wing length in Drosophila flies (Huey et al., 2000) and life-history traits in guppies (Reznick et al., 1997). Furthermore, the characteristics of a species' niche can have an important influence on the intensity of sexual selection and the evolution of sexual signals (Emlen \& Oring, 1977; Endler, 1992). For example, predation risk can constrain the expression of sexual signals (Endler, 1983), morphological feeding adaptations have been found to influence vocal performance, a trait used in mate choice (Ballentine, 2006), ambient light can drive the evolution of colour signals at a community scale (Gomez \& Théry, 2004), and a recent study found an association between habitat complexity and the presence of visual sexual traits (e.g. coloration, elongated fins) in Tanganyikan cichlids (Tsuboi et al., 2011). Hence, natural and sexual selection might be predicted to evolve in concert as invasion of new niches and adaptation to different ecological conditions open the door for evolution of distinct mating systems and new sexual signals (Emlen \& Oring, 1977; Endler, 1992; Andersson, 1994).

Here, we use recently developed phylogenetic comparative analyses to reconstruct the temporal dynamics of the evolution of traits associated with ecological adaptation and sexual selection in Tanganyikan cichlids. By analysing the rates of phenotypic evolution, we were able to test whether ecological characters and sexual traits evolve distinctly or through parallel temporal patterns, presenting similar rates of evolution during adaptive radiation. The Tanganyikan radiation is the oldest of the three African Great Lake cichlid radiations, including over 200 described species, and 250 or more endemic cichlid species are estimated to exist in the lake (Snoeks, 2000; Koblmü ller et al., 2008b). It represents the morphologically, behaviourally and ecologically most diverse cichlid assemblage (Chakrabarty, 2005; Koblmü ller et al., 2008b; Young et al., 2009) and is thus a suitable system for testing these questions.

\section{Methods}

\section{Phenotypic traits}

We selected phenotypic traits that mainly influenced (i) survival, and hence were under natural selection, or (ii) mating success, and thus either reflected the intensity of or were under sexual selection (Darwin, 1871). We used diet, habitat and depth to characterize a species' niche. These ecological traits have been previously shown to be associated with a suite of morphological adaptations (Bouton et al., 2002; Clabaut et al., 2007; Gonzalez-Voyer et al., 2009c; Salzburger, 2009) and should capture variation in a large number of distinct traits associated with ecological adaptation. Form of care (i.e. substrate guarding or mouthbrooding) was included as a behavioural trait under natural selection. As female body size reflects selection on both fecundity and propagule size in cichlid fishes and thus is strongly linked to fitness (Kolm et al., 2006a,b), we considered female size to represent a life-history trait under natural selection. To describe variation in the strength of sexual selection, we used mating system and prevalence of sperm competition as estimates of the intensity of pre- and post-copulatory sexual selection, and sexual size dimorphism (SSD), sexual dichromatism and sexual shape dimorphism as secondary sexual traits. Previous studies have shown that 

SSD, sexual dichromatism and sexual shape dimorphism are significantly correlated with mating system and prevalence of sperm competition in Tanganyikan cichlids (Gonzalez-Voyer et al., 2008; Tsuboi et al., 2011).

Diet and habitat were coded as continuous variables representing variation in prey motility and habitat complexity. Qualitative descriptions of both variables were transformed into quantitative continuous variables reflecting a continuum of variation. Diet reflected variation in prey motility, with sessile prey such as aufwuchs and fixed algae at one extreme and fishes at the other (for more details see the study by Gonzalez-Voyer et al., 2009c). Habitat reflected variation in complexity: benthic and benthopelagic habitats were the least complex and rocky habitats the most complex (for more details see the study by Gonzalez-Voyer et al., 2009c). It has been previously shown that such categorical ranking of habitats captures significant variation in quantitative measures of complexity (Pollen et al., 2007). Most species do not strictly inhabit a single habitat or feed on one prey type; therefore, we used descriptive information on habitat preferences and prey to calculate an average for each species giving more weight to preferred habitats/prey based on detailed descriptions from primary publications (see the study by Gonzalez-Voyer et al., 2009c). This approach has the added benefit of also capturing within species variation in prey and habitat preferences. Form of care was coded as a dichotomous variable representing mouthbrooding or substrate guarding (as in Gonzalez-Voyer et al., 2009c). Information on depth was collected from the study by Konings (2005), as well as from FishBase, and by contacting experts on particular species for which no published data were available. When a range of depths was provided, the median was used. For female body size, we used standard length (mm) as collected by Erlandsson \& Ribbink (1997) as well as from sexually mature specimens collected in the field (range 4-7 individuals per species). Sexual size dimorphism was calculated using the index of Lovich \& Gibbons (1992) based on standard length of male and female adult specimens (data in the study by Erlandsson \& Ribbink (1997) and collected in the field). Prevalence of sperm competition was ranked (1-4) based on the information about mating system and fertilization location, which have previously been found to correlate significantly with different sperm characteristics (Fitzpatrick et al., 2009). Mating system was coded as by Seehausen et al. (1999) to reflect intensity of precopulatory sexual selection. Ranks varied from 1 to 4 , although they are taken to reflect a continuum of variation in behavioural mating system, with monogamous species at one extreme and promiscuous species, e.g. lekking, at the other extreme. Note, however, that there is evidence suggesting multiple paternity may occur in some apparently socially monogamous species (Sefc et al., 2008). Sexual dichromatism and sexual shape dimorphism were ranked independently by four Tanganyikan cichlid experts. Sexual dichromatism represented conspicuous differences between the sexes in coloration, even if the difference was restricted to the mating period. Shape dimorphism referred to clearly distinguishable differences between the sexes in traits such as fins or humps, which were not only the result of differences in body size. Shape dimorphism, in this context, does not relate to potentially less conspicuous differences between the sexes, as could be obtained using morphometric measures for example, and which could reflect ecological adaptation as well as sexual selection. Rather, our interest focussed on categorizing traits potentially exaggerated as a result of sexual selection, such as elongated fins displayed by males during courtship [presence of such traits has been found to be associated with more promiscuous mating systems (Tsuboi et al., 2011)]. For each species, the judges were asked whether the sexes presented conspicuous differences in coloration or shape (independently of size dimorphism); both variables were coded as dichotomous reflecting the presence or absence of sexual differences. Disagreement between the experts was limited to the ranks for sexual shape dimorphism of four species. In these rare instances, we used the rank of the expert who had most experience in observing the species in their natural habitat ( $H$. Büscher). A table presenting trait values and list of species included in the study is available as online Appendix.

\section{Phylogenetic reconstruction}

We reconstructed a molecular phylogeny for the 49 cichlid species for which we were able to collect data on all traits of interest using mitochondrial sequences downloaded from GenBank. We used two coding sequences, cytochrome $\mathrm{b}$ and $\mathrm{NADH} 2$, and one noncoding gene, the control region. In some cases, we were obliged to combine genes from different studies (hence, different individuals or populations), because for a given species, the three genes were not available from the same study. To ensure that the concatenation of genes from different origins did not bias phylogenetic reconstruction, we first undertook single-gene analyses. Having confirmed phylogenetic congruence, the three genes were concatenated to create a matrix of 1819 base pairs. Because the comparative methods used here require an ultrametric phylogeny, we used the program BEAST (Drummond \& Rambaut, 2007) for phylogenetic inference. Analyses were run under a GTR $+\mathrm{I}+\mathrm{C}$ model of substitution selected using jModel test (Posada, 2008). We ran the analysis for 30 million iterations sampling the Markov chain every 1000 iterations. We used a log normal, relaxed molecular clock and set the mean rate of substitution to a fixed value of 1.0 (as recommended in the BEAST manual), because we had no external calibration points and our aim was not to estimate divergence times. Convergence was checked using Tracer v1.5, ensuring that the Markov chain had reached a 
stable likelihood value for all parameters; estimated sample sizes of all parameter values were $>480$ (ranges $=481-2679$ ). We obtained a single maximum clade credibility tree with a burnin of 7500 trees (see Supporting Information Fig. S1 for a figure showing the consensus tree).

Our phylogeny included species from 11 of the 16 tribes into which Tanganyikan cichlids have been classified (reviewed in Koblmü ller et al., 2008b), hence covering a large part of the lake's existing diversity. The reconstructed phylogeny was congruent with previous phylogenetic hypotheses (Salzburger et al., 2002).

\section{Rate of evolution analyses}

Phenotypic rates of evolution were analysed using two complementary methods (for the continuous traits): the disparity index (DI) (Harmon et al., 2003) and a comparison between the basic Brownian motion and OrnsteinUhlenbeck (OU) models of evolution, including the maximum-likelihood estimate of the alpha (a) parameter (Hansen, 1997; Martins \& Hansen, 1997; Butler \& King, 2004). Rate of phenotypic evolution for the discrete traits (form of care, sexual dichromatism and sexual shape dimorphism) was estimated based on transition rates between the two possible values of the trait under a continuous time Markov model of evolution (Pagel, 1999). Tempo of evolution comparisons between ecological characters and sexual traits was thus performed separately for continuous and discrete traits.

\section{Rate of evolution of continuous traits}

To examine the temporal patterns of phenotypic divergence, we calculated disparity through time plots (Harmon et al., 2003) for all continuous traits, using the package GEIGER (Harmon et al., 2008) in R (R Core Development Team, 2009). Disparity was calculated from average pair-wise Euclidean distances between species. Disparity through time was calculated as the average relative disparity of each subclade by dividing the average disparity of all subclades whose ancestral lineages were present at that time by the average disparity of the clade as a whole, and repeating this at each divergence event (i.e. each node) moving up the phylogeny from root to tip. A null hypothesis was constructed by simulating phenotypic divergence of each trait along the phylogeny under an unconstrained Brownian motion model and estimating disparity through time of the simulated trait (Harmon et al., 2003). The DI was calculated as the sum of the areas between the curve describing the phenotypic disparity of the trait and the curve describing the disparity under the null hypothesis of Brownian motion. Areas in which observed values were above expected were assigned positive values, whereas those below expected were assigned negative values. The DI thus describes how phenotypic disparity is partitioned along the phylogeny: values above 0 indicate that most phenotypic disparity is distributed within clades, suggesting that subclades include a large proportion of the overall disparity in the clade, negative values suggest that disparity is distributed among clades, suggesting early divergence, and values near 0 indicate that evolution has followed Brownian motion (Harmon et al., 2003; Gonzalez-Voyer et al., 2009b). Because we compare among traits within the same group of species, a trait with a high DI compared with another with a low DI suggests that closely related species differ more with respect to the former trait than with respect to the later. We calculated the DI comparing the relative disparity of the trait with each of the 1000 simulations of trait evolution under Brownian motion and obtained the mean of the sample and 95\% confidence intervals (CI) using nonparametric bootstrapping. By comparing whether the $95 \%$ CI of the mean DI overlapped with 0 (the expected mean value of the DI under the null model), we could obtain an estimate of the certainty with which the mean value of the DI differed from Brownian motion. To avoid confounding effects of incomplete species coverage in our phylogeny and overestimation of disparity between closely related species, we estimated disparity for only the first $4 / 5$ of the phylogeny (Harmon et al., 2003). For the ease of interpretation in the disparity through time plots, we present the timescale as million of years to the present, using 11 million years ago (MYA) as an estimate for the origin of the Tanganyikan cichlid radiation (Koblmü ller et al., 2008a). We would like to highlight that debate is ongoing regarding the age of the Tanganyikan cichlid radiation (see for example the study by Genner et al., 2007; Koblmü ller et al., 2008a; Schwarzer et al., 2009). However, here the timescale of the disparity through time plots is presented for illustration purposes only, and the results are the same independently of the root-age chosen for the Tanganyikan radiation.

Second, we calculated maximum-likelihood values for the a parameter, which is based on an OU process, and estimated the strength of selection acting on the trait (Hansen, 1997; Butler \& King, 2004), using the package GEIGER (Harmon et al., 2008) in R (R Core Development Team, 2009). The OU model is the simplest mathematical expression for an evolutionary model incorporating selection, and it differs from a Brownian model, in that it possesses a selective optimum (Butler \& King, 2004). The OU model has two terms:

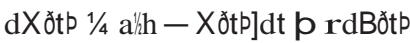

the first term describes change in character $X$ over the course of a small increment in time, the second term is random variation accumulating with time, or in other words a Brownian process (Butler \& King, 2004). The parameter a describes the strength of selection under an OU model, the higher the value of a, the stronger the selective regime; $\mathrm{h}$ is the value of the selective optimum (Butler \& King, 2004). Under an OU model, the rate of 
phenotypic change along the branches of a phylogeny depends on two things: (i) the distance between the actual trait value and the value of the selective optimum and (ii) the strength of the 'pull' towards the selective optimum, given by the value of a. Hence, a higher value of a indicates a stronger 'pull' towards the selective optimum. As a increases, the rate of phenotypic evolution along the branches of the tree will be increasingly faster, as compared to a basic Brownian process (Butler \& King, 2004; Gonzalez-Voyer et al., 2009a). When comparing between traits, the importance therefore lies in the value of a, the strength of the selection regime (Gonzalez-Voyer et al., 2009a). For each trait, we compared the fit of a Brownian motion model, where divergence accumulates gradually with time, with the fit of the OU model using a log-likelihood ratio test. We used the comparison between the two evolutionary models and estimates of a as a measure of the strength of selection acting on traits. We fitted an OU model with a single optimum, instead of more complex models with multiple optima because we had no a priori independent means of estimating potentially different selection regimes for each trait (Butler \& King, 2004). Hence, the value of a will provide an estimate of the tempo of evolution of the phenotypic trait.

\section{Rate of evolution of discrete traits}

For the discrete traits, we estimated the value of the transition parameter (q) under a continuous time Markov model of evolution (Pagel, 1999) using the package GEIGER (Harmon et al., 2008) in R (R Core Development Team, 2009). For all traits, we used a model in which all transitions were set to equal values because more complex models with unequal rates did not provide a significantly better fit than the simple model (results not shown). Mating system and sperm competition were also analysed as discrete traits, because they were codified as ranks even though they reflect a continuum of variation. This was performed to ensure that the tempo of evolution of these traits was independent of the method used to estimate it, because strictly speaking, the traits were categorical even though they reflected a continuum of variation.

\section{Estimates of error in the evolutionary parameters}

To estimate the error around the maximum-likelihood estimates of the evolutionary parameters [a and transition parameters (q)], we used a simulation-based approach. For each trait, we simulated evolution on our phylogeny using the maximum-likelihood estimates of the evolutionary parameters for the trait as generating conditions for the simulations in the package GEIGER (Harmon et al., 2008) in R (R Core Development Team, 2009). Hence, continuous traits were simulated to evolve under the model of evolution (Brownian motion or OU) found to provide a better fit for the trait. Simulations for each trait were performed using the variance-covariance matrix of the trait, and under the OU model, we used the maximumlikelihood estimate of a for the simulations. Discrete traits were simulated to evolve using the estimated value of $q$ for the transition matrix. We then estimated the values of the evolutionary parameters on 100 simulated data sets (for each trait) ensuring proper convergence was achieved. We used the median value as a measure of central tendency for the estimated evolutionary parameters from the simulations because the distributions were non-normal. As a measure of variance around the median, we calculated 95\% CI. Methods to calculate 95\% CI generally assume that the sample statistic follows a normal distribution, and when the distribution of the sample statistic is unknown or non-normal, it is preferable to use resampling methods to generate standard errors (Quinn \& Keough, 2002). Because the distributions of the simulated parameters were generally non-normal, we used nonparametric bootstrapping, which makes no assumptions about the distribution of the data, and a sampling frequency of 1000 (Manly, 1997; Quinn \& Keough, 2002). The simulations allowed us to include an estimate of variance around parameter values resulting from stochasticity in the simulations of trait evolution as well as to directly compare the rate of evolution between the traits of interest. The analyses described earlier, comparing between the null Brownian motion model and the OU model, indicated whether traits differed from the null model. By including these simulations, we are able to directly compare the tempo of evolution between traits. Two traits can be taken as presenting different rates of evolution if the 95\% CI of the medians of the parameter estimates do not overlap.

\section{Results}

\section{Rate of evolution of continuous traits}

\section{Disparity index}

Ecological characters and sexual traits presented notably different evolutionary patterns. The disparity indices of the sexual traits were more than one order of magnitude larger than those of the ecological characters and the lifehistory trait (see Table 1). Furthermore, the 95\% CI of the mean of the disparity indices for the ecological characters were all smaller than 0 and spanned 0 for the life-history trait. On the other hand, the 95\% CI of the mean disparity indices for all sexual traits were higher than 0. Hence, our results suggest that the disparity in ecological characters, and the life-history trait, is mainly concentrated among subclades, indicating that most divergence in these traits occurred early in the radiation, followed by gradual evolution as shown by the negative values of the disparity indices (see Table 1). Disparity in sexual traits, on the other hand, appears to be mainly concentrated within subclades, indicating that divergence occurred also at later stages in the radiation. The difference in the timing of divergence between ecological characters and sexual traits is apparent in the 
Table 1 Mean disparity indices and their 95\% CI (see Methods for details) for ecological characters, a life-history trait and sexual traits. The disparity index describes whether phenotypic differences among species originated early in the radiation (values $£ 0$ ) or result from recent divergence (values $>0$ ).

\begin{tabular}{lll}
\hline Trait & Disparity index & $95 \% \mathrm{Cl}$ \\
\hline $\begin{array}{ll}\text { Ecological characters } \\
\quad \text { Diet }\end{array}$ & 0.05 & ) 0.06 to $) 0.04$ \\
$\quad$ Habitat & 0.09 & ) 0.10 to $) 0.09$ \\
$\quad$ Depth & 0.12 & ) 0.12 to $) 0.11$ \\
$\begin{array}{l}\text { Life-history trait } \\
\quad \text { Female body size }\end{array}$ & \\
$\begin{array}{l}\text { Sexual traits } \\
\quad \text { Sexual size dimorphism }\end{array}$ & 0.002 & ) 0.006 to 0.009 \\
$\quad$ Sperm competition & 0.33 & \\
$\quad$ Mating system & 0.10 & 0.15 to 0.16 \\
\end{tabular}

diversity through time plots shown in Fig. 1. These plots were generated by running separate analyses of the pooled ecological characters (including female body size) on the one hand and the pooled sexual traits on the other. The plots show that relative disparity in sexual traits remains high throughout the radiation, even at the later stages, in contrast to the pattern in the ecological characters. The difference in the disparity indices also suggests different patterns of evolution as the pooled sexual traits presented a DI more than twice as large as that of the pooled ecological characters (see Fig. 1). Interestingly, both ecological characters and sexual traits present a recent increase in relative disparity occurring roughly at the same time, ca 4 MYA, although it is not as marked in the ecological characters as it is in the sexual traits.

\section{Estimates of the strength of selection}

The value of a for SSD, and the prevalence of sperm competition, was nearly twice the value of any ecological character, indicating stronger selection acting on these two sexual traits in comparison with the ecological characters and the life-history trait (Table 2). However, for mating system, the estimate of a was actually lower than the estimates for the ecological characters or the life-history trait (see Table 2). The OU model explained the evolution of SSD and prevalence of sperm competition significantly better than a Brownian model (Table 2). For the ecological characters and life-history trait, the OU model provided a significantly better fit than the Brownian model for diet and habitat, although the values of a were lower than those of SSD and sperm competition (Table 2).

Results from simulations indicate that the variance around the median value of the estimated alpha parameters that is due to the stochastic nature of evolution is rather small, providing further support for the differences in the rate of evolution of the sexual traits as compared with the ecological and life-history characters. Traits

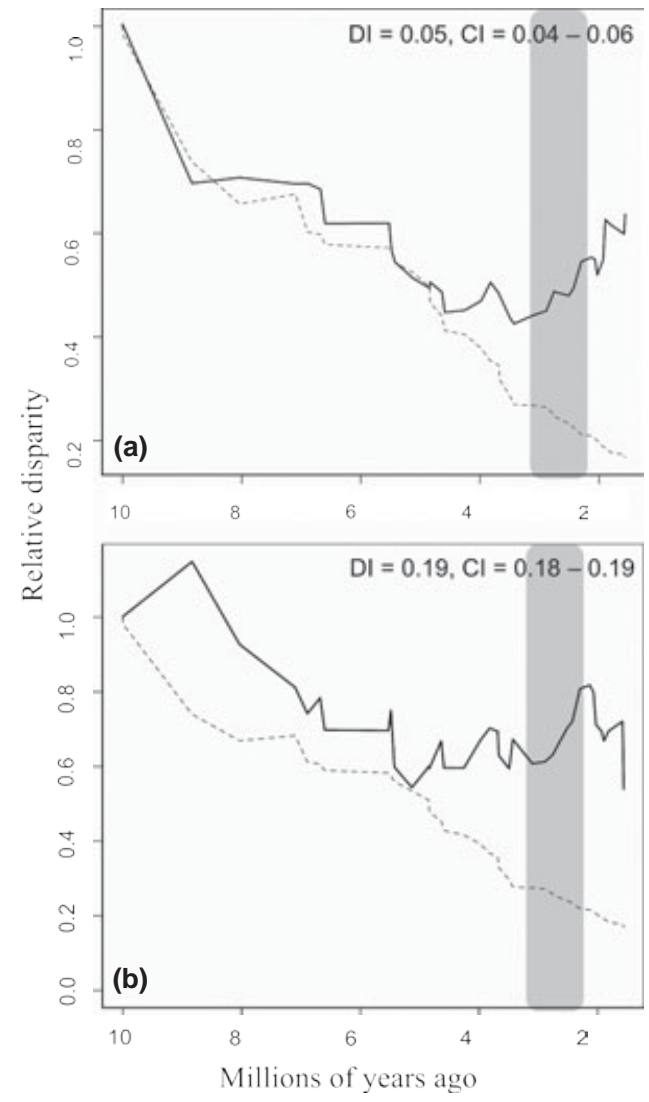

Fig. 1 Disparity through time plots for the pooled ecological characters and the life-history trait (a), and pooled sexual traits (b), including their respective average disparity indices and 95\% CI. For ease of interpretation, the $\mathrm{x}$-axis presents time to the present, taking 11 MYA as the date of initiation of the Tanganyikan radiation (see Methods). The bold black line shows the relative disparity for the pooled ecological characters and life-history trait (in a) and the pooled sexual traits (in b), and the dashed line shows the relative disparity of the traits simulated as evolving under Brownian motion (see Methods for details). The grey box highlights the age at which the 'secondary' Tanganyikan radiation is estimated to have occurred (ca. 3-2.5 MYA).

evolved under starting conditions (variance-covariance matrix and maximum-likelihood model of phenotypic evolution) similar to those of each of the ecological characters, and the life-history trait presented notably lower median values of a than those of traits simulated to evolve under starting conditions similar to those of SSD and sperm competition (see Fig. 2). Even diet and habitat, for which simulation of trait evolution was performed under an OU model, presented, nonetheless, notably lower median a values than those of the two sexual traits (see Fig. 2). As can be seen in Fig. 2, the $95 \%$ CI of the median a values for SSD and prevalence of sperm competition do not overlap with those of any of the ecological characters or with that of the life-history trait, indicating that their median values are different. 
Table 2 Estimates of the tempo of evolution of ecological characters, a life-history trait and sexual traits. Shown are the loglikelihood values of the two models of phenotypic evolution, Brownian motion and the Ornstein-Uhlenbeck (OU) model. The alpha parameter of the OU model describes the strength of selection acting on a trait; higher values indicate faster evolution. The two models of evolution are compared via a log-likelihood ratio test, and the table presents the $\mathrm{P}$-value of the test (significant differences are shown in bold).

\begin{tabular}{|c|c|c|c|c|}
\hline \multirow[b]{2}{*}{ Trait } & \multirow{2}{*}{$\begin{array}{l}\text { Brownian motion } \\
\text { Log likelihood }\end{array}$} & \multicolumn{3}{|l|}{ Alpha } \\
\hline & & a value & $\begin{array}{l}\text { Log } \\
\text { likelihood }\end{array}$ & $\mathrm{P}$ \\
\hline \multicolumn{5}{|l|}{ Ecological characters } \\
\hline Diet & ) 81.62 & 14.7 & ) 77.26 & 0.003 \\
\hline Habitat & ) 75.32 & 10.4 & ) 72.66 & 0.02 \\
\hline Depth & )23.23 & 7.9 & ) 21.65 & 0.08 \\
\hline \multicolumn{5}{|l|}{ Life-history trait } \\
\hline Female body size & 18.43 & 12.3 & 19.95 & 0.08 \\
\hline \multicolumn{5}{|l|}{ Sexual traits } \\
\hline Sexual size dimorphism & 59.14 & 25.6 & 65.74 & 0.0003 \\
\hline Sperm competition & ) 72.62 & 27.8 & $\longdiv { 6 6 . 3 4 }$ & 0.0004 \\
\hline Mating system & 71.49 & 4.1 & 71.09 & 0.37 \\
\hline
\end{tabular}

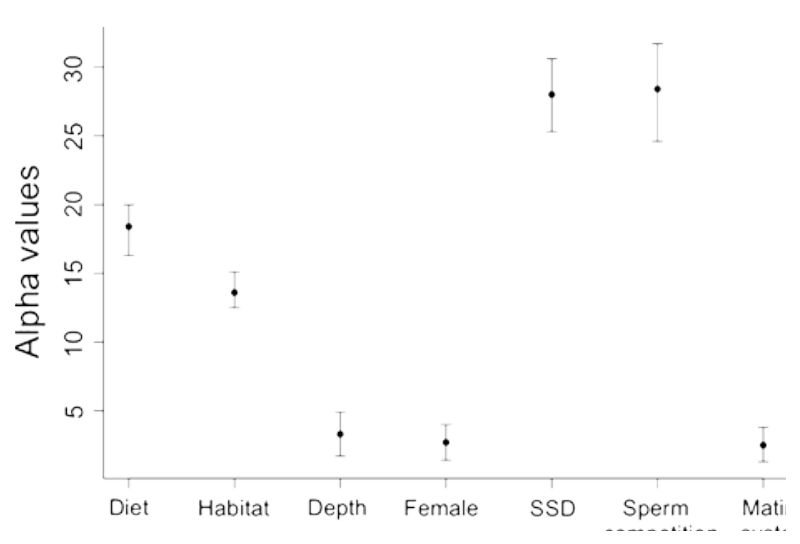

Fig. 2 Median alpha values and their 95\% CI for traits simulated to evolve on the phylogeny under different conditions. Diet and habitat were simulated under an OU model with alpha $=14.7$ and 10.4 , respectively (see Results for details). Depth and female (female standard length) were simulated under a Brownian motion model, as was mating system. Finally, sexual size dimorphism (SSD) and sperm (prevalence of sperm competition) were simulated under an OU model with alpha $=25.6$ and 27.8, respectively. Black circles show the median value of the parameter estimates, and the bars show the $95 \%$ CI of the median.

\section{Rate of evolution of discrete traits}

The analyses of discrete traits support the results for the continuous traits. The two discrete sexual traits (sexual dichromatism and sexual shape dimorphism) presented much higher transition rates (5.18 and 2.24, respectively) than the discrete naturally selected trait (form of care,
0.40). Mating system and sperm competition also presented high transition rates when analysed as discrete traits under a Markov model (16.04 and 9.27, respectively). These results thus also indicate that sexual traits present a higher rate of evolution than naturally selected traits.

As described earlier, rates of transition estimated for discrete traits from data evolved under starting conditions identical to those estimated for sexual traits (dichromatism and shape dimorphism) were higher than trait parameters evolved under starting conditions identical to those of a naturally selected trait (form of care) (medians and their 95\% CI: 5.15, 4.65-5.75; 2.20, 1.862.49; and $0.39,0.39-0.40$, respectively). Also, when we simulated trait evolution under starting conditions identical to those of sperm competition and mating system (analysed as discrete traits), the parameters were notably higher than those of traits evolved under conditions identical to those of sexual dichromatism (medians and their 95\% CI: sperm competition $=55.61$, 46.21-63.18; mating system $=20.85,18.87-22.87)$. The difference could be due, in part, to the fact that sperm competition and mating system were traits with four states (rather than the two states of the other discrete traits), and hence higher opportunities for evolutionary transitions to occur. Even so, the striking differences, and nonoverlapping CI with those of form of care, lend support to a faster tempo of evolution in sperm competition and mating system.

\section{Discussion}

Our results show that in Tanganyikan cichlids traits under natural selection present different evolutionary patterns than sexual traits and further that sexual traits have a faster tempo of evolution than ecological characters or life-history traits also during adaptive radiation. Our results thus support verbal arguments, formal mathematical models and available empirical evidence all of which suggest that sexually selected traits exhibit faster evolutionary rates than naturally selected traits (West-Eberhard, 1983; Arnqvist, 1998; Holland \& Rice, 1998; Gavrilets, 2000; Chapman et al., 2003). Further, a recent meta-analysis of measures of the strength of phenotypic selection in natural populations found that traits influencing mating success were under stronger selection than traits related with survival (Kingsolver et al., 2001). Our results also provide empirical evidence indicating that faster phenotypic evolution of sexual traits occurs during adaptive radiation, at least in Tanganyikan cichlids. Interestingly, our results suggest that different traits, some of which may be influenced by an interaction between ecological characteristics of a species' niche and sexual selection (Endler, 1992), nonetheless, may present distinct evolutionary patterns from purely ecological characters, under natural selection. Indeed, results from a previous study with 
Tanganyikan cichlids suggest that even highly correlated phenotypic traits can show distinct evolutionary patterns (Gonzalez-Voyer et al., 2009b).

Mating system was the only trait to show somewhat contradictory results. The DI was higher than for any of the naturally selected traits, although lower than for SSD or prevalence of sperm competition. The maximumlikelihood estimate of a, however, was actually lower than that of the ecological characters, although when analysed as a discrete trait, the transition values (q) were higher than for the naturally selected trait (form of care). It is possible that our measure did not capture the complexity of the cichlid mating systems. However, because cichlids apparently have a highly conserved courtship behaviour across lineages (Stelkens et al., 2010), it is unlikely we are missing variation in such behavioural traits. On the other hand, there can be some discrepancy between the behavioural mating system and what is observed using genetic markers, as some monogamous species show high levels of extra-pair paternity, and among-population differences in mating system have been observed in one species (Sefc et al., 2008, 2009). It is also possible that environmental factors constrained the evolution of mating system (Emlen \& Oring, 1977). Indeed, in substrate guarding Tanganyikan cichlids, the availability of suitable spawning sites within a male's territory determines the degree of polygyny. Polygynous males defend territories where several females lay their eggs and care for the brood alone, whereas monogamous males defend territories with a single spawning female and provide some parental care (Gashagaza, 1991). Such interactions between ecology and sexual selection might explain why mating system presented higher disparity indices, but not stronger selection, under an OU model, than did naturally selected traits.

A recent study compared phenotypic evolutionary patterns of body size and body shape across a wide diversity of adaptive radiations to test whether traits presented a burst of evolutionary divergence early in adaptive radiation, as predicted by theory (Harmon et al., 2010). Harmon et al. (2010) found that an OU model explained the evolution of body size in Tanganyikan cichlids better than a model including an early burst of evolution. However, we found that a Brownian model explained the evolution of female body size better. The difference between our results and those obtained by Harmon et al. (2010) may be due to the fact that their measure of body size combined information from both males and females. As we have shown here, SSD is best explained by an OU model, and hence, it is possible that species-specific body size reflects the combined action of both natural and sexual selection.

Might the observed rapid evolution towards the optimum of the sexual characters explain the high DI values for these traits? Under an OU model, rapid phenotypic evolution of a trait can result in erosion of the phylogenetic signal, as the position of a lineage in phenotypic space becomes increasingly influenced by the position of the optimum rather than by shared ancestry (Revell et al., 2008). In an extreme case, if there were no phylogenetic signal in the data (sister species are no more similar for a given trait than any of them is to a random, more distantly related individual from the sample), then disparity within subclades will be a random sample of the total disparity, and the DI will be high. Such an explanation is unlikely for our sample as the values of $\mathrm{k}$ (Freckleton et al., 2002), a measure of phylogenetic signal, estimated for the continuous traits suggest that all of these, with the exception of SSD and sperm competition, presented significant phylogenetic signal, that is, that the $\mathrm{k}$ value was significantly different from 0 ( $\mathrm{k}$ range $=0.64-0.81$, results not shown). For SSD and sperm competition, the maximum-likelihood estimates of $\mathrm{k}$ were intermediate although not significantly different from $0 \quad\left(k=0.29, v^{2}=0.66, P=0.42\right.$, and $k=0.48, v^{2}=2.68, P=0.10$, respectively). Furthermore, when analysed as a continuous variable, mating system did not present significant support for rapid evolution under an OU model. However, mating system still presented a MDI value that was much higher than that of any ecological character and closer to values of the other sexual traits. Finally, SSD and sperm competition presented a clear temporal signal in relative disparity (results not shown) even though their DI was high, which would not be expected if the phylogenetic signal had been eroded.

Our results also suggest that sexual selection might have played an important role in recent divergence in Lake Tanganyika. As predicted by theory and adaptive radiation models (Gavrilets, 2000; Schluter, 2000; Danley \& Kocher, 2001), traits associated with ecological adaptation showed early divergence, with disparity mainly concentrated among subclades, followed by gradual evolution. Still, the disparity through time plot for the combined ecological characters, and the life-history trait, does point to a recent upsurge in disparity, with the curve for trait disparity diverging from the Brownian simulations at about 4 MYA. Based on morphological characteristics, the Tanganyikan cichlids have been classified into 16 tribes (Takahashi, 2003), which are largely supported by molecular data (Koblmü ller et al., 2008b). The suggestion that the primary Tanganyikan radiation was mainly driven by natural selection received support from observations that most tribes are limited to particular ecological niches (Koblmü ller et al., 2008b). Early divergence based on adaptation to macrohabitat characteristics appears to be common in cichlid radiations (see for example the study by Nagl et al., 2000; Danley \& Kocher, 2001). A similar pattern as that in the African cichlids is observed in Anolis lizards of the Greater Antilles, where multiple ecomorphs associated with separate habitats have evolved repeatedly (Losos et al., 1998), as well as in three-spine sticklebacks which diverged into distinct limnetic and benthic forms in multiple North-American Lakes (Schluter, 2000). Most 
ecological characters, with the exception of diet and habitat, also showed evidence of gradual evolution, with change accumulating with time, as was the case for female body size. Furthermore, the maximum-likelihood estimates of a for all ecological characters and the life-history trait were notably lower than for sperm competition and SSD, and the 95\% CI of the median a values from the simulations did not overlap. Note that this is not to say that traits associated with ecological adaptation were not under strong selection at the early stages of the radiation, but rather that currently, Tanganyikan cichlids do not show evidence of strong disruptive natural selection.

Debate continues regarding the ages of the African cichlid radiations. For Lake Tanganyika, the cichlid radiation has been estimated to originate at $22.7 \pm 3.5$, $20 \pm 6,10.4 \pm 1.6$ and $6-5$ MYA depending on the data used for calibrations (Genner et al., 2007; Koblmü ller et al., 2008a; Schwarzer et al., 2009). At this point, several lineages of cichlids independently colonized the emerging lake, seeding the so-called primary lacustrine radiation at which time the substrate breeding Lamprologini and mouthbrooding C-lineage diversified rapidly into several lineages (reviewed in Koblmü ller et al., 2008b). A 'secondary radiation', involving most mouthbrooding lineages, apparently took place at about half the age of the primary radiation (Salzburger et al., 2005; Koblmü ller et al., 2008b), in Fig. 1 shown to have occurred ca. 2.5-3 MYA [although based on an origin of 16 MYA for the Tanganyikan radiation, the 'secondary radiation' would have occurred ca. 8-6 MYA (see the study by Genner et al., 2007; Koblmü ller et al., 2008a,b)]. Our results are in accord with previous observations suggesting that the primary radiation was primarily driven by divergence based on ecological adaptation (Koblmü ller et al., 2008b). The 'secondary radiation' (area highlighted in grey in Fig. 1) appears to have been accompanied by an upsurge in disparity in both ecological characters and sexual traits. The increase in relative disparity during the secondary radiation, however, is higher for the sexual traits: compare the peak in relative disparity at ca. 3 MYA for the ecological characters $(=0.5)$ to the peak at roughly the same time for the sexual traits $(=0.9)$. We cannot rule out that ecological divergence could have been mainly the result of fine niche partitioning based on refinement of the trophic apparatus, involving a narrow scope of morphological adaptations that our data might not have allowed us to detect (Danley \& Kocher, 2001). However, the high relative disparity observed in sexually selected traits, even at ca. 3-2.5 MYA (see Fig. 1b), suggests it is possible that divergence based on sexually selected traits was an important driver of the secondary radiation. This suggestion could be further investigated using more complete species sampling and a higher diversity of secondary sexual traits.

In conclusion, we suggest that sexual selection has played an important role in generating recent diver- gence during the Tanganyikan cichlid radiation. Further, although sexual traits may be influenced by characteristics of a species' niche, they still present high disparity throughout the radiation and higher tempo of evolution than ecological characters and life-history traits. Our results thus suggest that sexual selection may be able to continue to generate divergence even after ecological specialization (Seehausen, 2000). A possible avenue for future research is to test whether a higher propensity for sexual selection at later stages of an adaptive radiation also is associated with higher species richness.

\section{Acknowledgments}

The work was funded by Wenner-Grens Foundations and Swedish Research Council grants to N. Kolm. A. Gonzalez-Voyer was funded by a Wenner-Grens Foundations post-doctoral stipend and a Juan de la Cierva postdoctoral contract from the Spanish Ministry of Science and Innovation. Heinz S. Büscher, John L. Fitzpatrick, Ola Svensson, Carl Westholm and S. Koblmü ller assisted with species ranking. Members of the r-sig-phylo mailing list provided useful answers to questions about data analysis and simulations. Associate Editor A. Caballero and two reviewers provided valuable comments.

\section{References}

Albertson, R.C., Markert, J.A., Danley, P.D. \& Kocher, T.D. 1999. Phylogeny of a rapidly evolving clade: the cichlid fishes of Lake Malawi, East Africa. Proc. Natl. Acad. Sci. USA. 96: 5107-5110.

Andersson, M. 1994. Sexual Selection. Princeton University Press, Princeton, N J.

Arnqvist, G. 1998. Comparative evidence for the evolution of genitalia by sexual selection. Nature 393: 784-786.

Ballentine, B. 2006. Morphological adpatation influences the evolution of a mating signal. Evolution 60: 1936-1944.

Barlow, G.W. 2000. The Cichlid Fishes: Nature's Grand Experiment in Evolution. Perseus Publishing, Cambridge, MA.

Boake, C.R.B. 2005. Sexual selection and speciation in Hawaiian Drosophila. Behav. Genet. 35: 297-303.

Bouton, N., de Visser, J. \& Barel, C.D.N. 2002. Correlating head shape with ecological variables in rock-dwelling haplochromines (Teleostei: Cichlidae) from Lake Victoria. Biol. J. Linn. Soc. 76: 39-48.

Butler, M.A. \& King, A.A. 2004. Phylogenetic comparative analysis: a modeling approach for adaptive evolution. Am. Nat. 164: 683-695.

Chakrabarty, P. 2005. Testing conjectures about morphological diversity in cichlids of lakes Malawi and Tanganyika. Copeia 2: 359-373.

Chapman, T., Arnqvist, G., Bangham, J. \& Rowe, L. 2003. Sexual conflict. Trends Ecol. Evol. 18: 41-47.

Clabaut, C., Bunje, P.M.E., Salzburger, W. \& Meyer, A. 2007. Geometric morphometric analyses provide evidence for the adaptive character of the Tanganyikan cichlid fish radiations. Evolution 61: 560-578. 
Crapon de Caprona, M.-D. 1986. Are preferences and tolerances in cichlid mating important for speciation? J. Fish Biol. 29: $151-158$.

Danley, P.D. \& Kocher, T.D. 2001. Speciation in rapidly diverging systems: lessons from Lake Malawi. Mol. Ecol. 10: 1075-1086.

Darwin, C. 1871. The Descent of Man, and Selection in Relation to Sex. J. Murray, London.

Deutsch, J.C. 1997. Colour diversification in Malawi cichlids: evidence for adaptation, reinforcement or sexual selection? Biol. J. Linn. Soc. 62: 1-14.

Drummond, A.J. \& Rambaut, A. 2007. BEAST: Bayesian evolutionary analysis by sampling trees. BMC Evol. Biol. 7: 8 .

Emlen, S.T. \& Oring, L.W. 1977. Ecology, sexual selection, and the evolution of mating systems. Science 197: 215-223.

Endler, J.A. 1983. Natural and sexual selection on color patterns in Poeciliid fishes. Environ. Biol. Fish 9: 173-190.

Endler, J.A. 1992. Signals, signal conditions, and the direction of evolution. Am. Nat. 139: S125-S153.

Erlandsson, A. \& Ribbink, A.J. 1997. Patterns of sexual size dimorphism in African cichlids. S. Afr. J. Sci. 93: 498-508.

Fisher, R.A. 1930. The Genetical Theory of Natural Selection. Clarendon Press, Oxford, UK.

Fitzpatrick, J.L., Montgomerie, R., Desjardins, J.K., Stiver, K.A., Kolm, N. \& Balshine, S. 2009. Female promiscuity promotes the evolution of faster sperm in cichlid fishes. Proc. Natl. Acad. Sci. USA. 106: 1128-1132.

Foster, D.J., Podos, J. \& Hendry, A.P. 2007. A geometric morphometric appraisal of beak shape in Darwin's finches. J. Evol. Biol. 21: 263-275.

Freckleton, R.P., Harvey, P.H. \& Pagel, M. 2002. Phylogenetic analysis and comparative data: a test and review of evidence. Am. Nat. 160: 712-726.

Gashagaza, M.M. 1991. Diversity of breeding habits in Lamprologine cichlids in Lake Tanganyika. Physiol. Ecol. Jpn. 28: 2965.

Gavrilets, S. 2000. Rapid evolution of reproductive barriers driven by sexual conflict. Nature 403: 886-889.

Gavrilets, S. \& Losos, J.B. 2009. Adaptive radiation: contrasting theory with data. Science 323: 732-737.

Genner, M.J., Seehausen, O., Lunt, D.H., Joyce, D.A., Shaw, P.W., Carvalho, G.R. et al. 2007. Age of cichlids: new dates for ancient lake fish radiations. Mol. Biol. Evol. 24: 1269-1282.

Gomez, D. \& Théry, M. 2004. Influence of ambient light on the evolution of colour signals: comparative analysis of a Neotropical rainforest bird community. Ecol. Lett. 7: 279284.

Gonzalez-Voyer, A., Fitzpatrick, J.L. \& Kolm, N. 2008. Sexual selection determines parental care patterns in cichlid fishes. Evolution 62: 2015-2026.

Gonzalez-Voyer, A., Winberg, S. \& Kolm, N. 2009a. Brain structure evolution in a basal vertebrate clade: evidence from phylogenetic comparative analysis of cichlid fishes. BMC Evol. Biol. 9: 238.

Gonzalez-Voyer, A., Winberg, S. \& Kolm, N. 2009b. Distinct evolutionary patterns of brain and body size during adaptive radiation. Evolution 63: 2266-2274.

Gonzalez-Voyer, A., Winberg, S. \& Kolm, N. 2009c. Social fishes and single mothers: brain evolution in African cichlids. Proc. Biol. Sci. 276: 161-167.

Grant, P.R. \& Grant, B.R. 2002. Unpredictable evolution in a 30year study of Darwin's finches. Science 296: 707-711.
Hansen, T.F. 1997. Stabilizing selection and the comparative analysis of adaptation. Evolution 51: 1341-1351.

Harmon, L.J., Schulte, J.A. II, Larson, A. \& Losos, J.B. 2003. Tempo and mode of evolutionary radiation in iguanian lizards. Science 301: 961-964.

Harmon, L.J., Weir, J.T., Brock, C.D., Glor, R.E. \& Challenger, W. 2008. GEIGER: investigating evolutionary radiations. Bioinformatics 24: 129-131.

Harmon, L.J., Losos, J.B., Davies, J.T., Gillespie, R.G., Gittleman, J.L., Jennings, W.B. et al. 2010. Early bursts of body size and shape evolution are rare in comparative data. Evolution 64: 2385-2396.

Holland, B. \& Rice, W.R. 1998. Chase-away sexual selection: antagonistic seduction versus resistance. Evolution 52: 1-7.

Huey, R.B., Gilchrist, G.W., Carlson, M.L., Berrigan, D. \& Serra, L. 2000. Rapid evolution of a geographic cline in size in an introduced fly. Science 287: 308-309.

Hulsey, C.D., García de León, F.J. \& Rodiles-Hernández, R. 2006. Micro- and macroevolutionary decoupling of cichlid jaws: a test of Liem's key innovation hypothesis. Evolution 60: 20962109.

Kingsolver, J.G., Hoekstra, H.E., Hoekstra, J.M., Berrigan, D., Vignieri, S.N., Hill, C.E. et al. 2001. The strength of phenotypic selection in natural populations. Am. Nat. 157: 245-261.

Koblmü ller, S., Schliewen, U.K., Duftner, N., Sefc, K.M., Katongo, C. \& Sturmbauer, C. 2008a. Age and spread of the haplochromine cichlid fishes in Africa. Mol. Phylogenet. Evol. 49: 153-169.

Koblmü ller, S., Sefc, K.M. \& Sturmbauer, C. 2008b. The lake Tanganyika cichlid species assemblage: recent advances in molecular phylogenetics. Hydrobiologia 615: 5-20.

Kolm, N., Goodwin, N.B., Balshine, S. \& Reynolds, J.D. 2006a. Life history evolution in cichlids 1: revisiting the evolution of life histories in relation to parental care. J. Evol. Biol. 19: 6675.

Kolm, N., Goodwin, N.B., Balshine, S. \& Reynolds, J.D. 2006b. Life history evolution in cichlids 2: directional evolution of the trade-off between egg number and egg size. J. Evol. Biol. 19: 76-84.

Konings, A. 2005. Back to Nature Guide to Tanganyika Cichlids, 2nd edn. Cichlid Press, El Paso, TX.

Liem, K. 1973. Evolutionary strategies and morphological innovations: cichlid pharyngeal jaws. Syst. Zool. 22: 425-441.

Losos, J.B. \& Miles, D.B. 2002. Testing the hypothesis that a clade has adaptively radiated: iguanid lizard clades as a case study. Am. Nat. 160: 147-157.

Losos, J.B., Jackman, T.R., Larson, A., de Queiroz, K. \& Rodriguez-Schettino, L. 1998. Contingency and determinism in replicated adaptive radiations of island lizards. Science 279: 2115-2118.

Lovich, J.E. \& Gibbons, J.W. 1992. A review of techniques for quantifying sexual size dimorphism. Growth Dev. Aging 56: 269-281.

Mabuchi, K., Miya, M., Azuma, Y. \& Nishida, M. 2007. Independent evolution of the specialized pharyngeal jaw apparatus in cichlid and labrid fishes. BMC Evol. Biol. 7.

Manly, B.F.J. 1997. Randomization and Monte Carlo Methods in Biology, 2nd edn. Chapman \& Hall/CRC, Boca Raton, Florida.

Martins, E.P. \& Hansen, T.F. 1997. Phylogenies and the comparative method: a general approach to incorporating phylogenetic information into the analysis of intespecific data. Am. Nat. 149: 646-667. 
Nagl, S., Tichy, H., Mayer, W.E., Takezaki, N., Takahata, N. \& Klein, J. 2000. The origin and age of haplochromine fishes in Lake Victoria, East Africa. Proc. Biol. Sci. 267: 1049-1061.

Pagel, M. 1999. Inferring the historical patterns of biological evolution. Nature 401: 877-884.

Pollen, A.A., Dobberfuhl, A.P., Scace, J., Igulu, M.M., Renn, S.C.P., Shumway, C.A. et al. 2007. Environmental complexity and social organization sculpt the brain in lake Tanganyikan cichlid fish. Brain Behav. Evol. 70: 21-39.

Posada, D. 2008. jModelTest: phylogenetic model averaging. Mol. Biol. Evol. 25: 1253-1256.

Quinn, G.P. \& Keough, M.J. 2002. Experimental Design and Data Analysis for Biologists. Cambridge University Press, Cambridge.

Revell, L.J., Harmon, L.J. \& Collar, D.C. 2008. Phylogenetic signal, evolutionary process and rate. Syst. Biol. 57: 591-601.

Reznick, D.N., Shaw, F.H., Rodd, F.H. \& Shaw, R.G. 1997. Evaluation of the rate of evolution in natural populations of guppies (Poecilia reticulata). Science 275: 1934-1937.

Salzburger, W. 2009. The interaction of sexually and naturally selected traits in the adaptive radiations of cichlid fishes. Mol. Ecol. 18: 169-185.

Salzburger, W., Meyer, A., Baric, E., Verheyen, E. \& Strumbauer, C. 2002. Phylogeny of Lake Tanganyika cichlid species flock and its relationship to the central and east African haplocrhomine cichlid fish faunas. Syst. Biol. 51: 113-135.

Salzburger, W., Mack, T., Verheyen, E. \& Meyer, A. 2005. Out of Tanganyika: genesis, explosive speciation, key-innovations and phylogeography of the haplochromine cichlid fishes. BMC Evol. Biol. 5: doi: 10.1186/1471-2148-5-17

Schluter, D. 2000. The Ecology of Adaptive Radiation. Oxford University Press, Oxford.

Schwarzer, J., Misof, B., Tautz, D. \& Schliewen, U.K. 2009. The root of the East African cichlid radiations. BMC Evol. Biol. 9.

Seehausen, O. 2000. Explosive speciation rates and unusual species richness in haplochromine cichlid fishes: effects of sexual selection. Adv. Ecol. Res. 31: 237-274.

Seehausen, O. \& Van Alphen, J.J.M. 1999. Can sympatric speciation by disruptive sexual selection explain rapid evolution of cichlid diversity in Lake Victoria. Ecol. Lett. 2: 262-271.

Seehausen, O., van Alphen, J.J.M. \& Witte, F. 1997. Cichlid fish diversity threatened by eutrophication that curbs sexual selection. Science 277: 1808-1811.

Seehausen, O., Mayhew, P.J. \& Van Alphen, J.J.M. 1999. Evolution of colour patterns in East African cichlid fish. J. Evol. Biol. 12: 514-534.

Seehausen, O., Terai, Y., Magalhaes, I.S., Carleton, K.L., Mrosso, H.D.J., Miyagi, R. et al. 2008. Speciation through sensory drive in cichlid fish. Nature 455: 620-626.

Sefc, K.M., Mattersdorfer, K., Sturmbauer, C. \& Koblmuller, S. 2008. High frequency of multiple paternity in broods of a socially monogamous cichlid fish with biparental nest defence. Mol. Ecol. 17: 2531-2543.

Sefc, K.M., Hermann, C.M. \& Koblmuller, S. 2009. Mating system variability in a mouthbrooding cichlid fish from a tropical lake. Mol. Ecol. 18: 3508-3517.

Simpson, G.G. 1953. The Major Features of Evolution. Columbia University Press, New York.

Snoeks, J. 2000. How well known is the ichthyodiversity of the large East African lakes? Adv. Ecol. Res. 31: 17-38.

Stelkens, R.B., Young, K.A. \& Seehausen, O. 2010. The accumulation of reproductive incompatibilities in African cichlid fish. Evolution 64: 617-633.

Takahashi, T. 2003. Systematics of Tanganyikan cichlid fishes (Teleostei: Perciformes). Ichthyol. Res. 50: 367-382.

R Core Development Team 2009. R: A Language and Environment for Statistical Computing. R Foundation for Statistical Computing, Vienna, Austria.

Tsuboi, M., Gonzalez-Voyer, A., Höglund, J. \& Kolm, N. 2011. The link between ecology and evolution of different types of sexual dimorphism in Tanganyikan cichlid fishes. Evol. Ecol.

West-Eberhard, M.J. 1983. Sexual selection, social competition, and speciation. Q. Rev. Biol. 58: 155-183.

Young, K.A., Snoeks, J. \& Seehausen, O. 2009. Morphological diversity and the roles of contingency, chance and determinism in African cichlid radiations. PLoS One 4: e4740.

\section{Supporting information}

Additional Supporting Information may be found in the online version of this article:

Appendix S1 Trait values for the four ecological characters, one life-history trait and five sexual traits included in the analyses, as well as the list of 49 species. Appendix S2 Accession numbers for the three mitochondrial genes used to construct the phylogeny.

Figure S1 Consensus phylogenetic tree obtained from the Bayesian reconstruction in BEAST based on a concatenated matrix of three mitochondrial sequences.

As a service to our authors and readers, this journal provides supporting information supplied by the authors. Such materials are peer-reviewed and may be reorganized for online delivery, but are not copy-edited or typeset. Technical support issues arising from supporting information (other than missing files) should be addressed to the authors.

Received 11 February 2011; revised 8 July 2011; accepted 10 July 2011 